\title{
Identification of a virulence-related surface protein XF in piscine Streptococcus agalactiae by pre-absorbed immunoproteomics
}

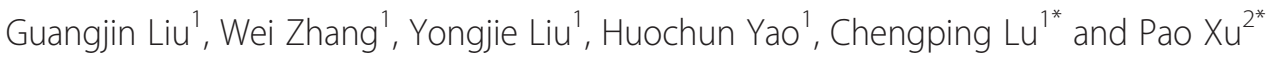

\begin{abstract}
Background: Since 2009, large-scale Streptococcus agalactiae infections have broken out in cultured tilapia farms in China, resulting in considerable economic losses. Screening of the surface proteins is required to identify virulence factors or protective antigens involved in piscine S.agalactiae infections in tilapia. Pre-absorbed immunoproteomics method (PAIM) is a useful method previously established in our laboratory for identifying bacterial surface proteins.

Results: A serine-rich repeat protein family 1 (Srr-1), designated XF, was identified by PAIM in piscine S. agalactiae isolate GD201008-001. To investigate the role of XF in the pathogenesis of piscine S. agalactiae, an isogenic $x f$ mutant strain $(\Delta x f)$ and a complemented strain $(C \Delta x f)$ were successfully constructed. The $\Delta x f$ mutant and $C \Delta x f$ showed no significant differences in growth characteristics and adherence to HEp-2 cells compared with the wild-type strain. However the $50 \%$ lethal dose of $\Delta x f$ was increased (4-fold) compared with that of the parental strain in a zebrafish infection model.
\end{abstract}

Conclusions: The findings demonstrated that XF is a virulence-related, highly immunoreactive surface protein and is involved in the pathogenicity of S. agalactiae infections in fish.

Keywords: Streptococcus agalactiae, Piscine, Pre-absorbed immunoproteomic method (PAIM), Serine-rich repeat protein (Srr), Zebrafish

\section{Background}

Streptococcus agalactiae, also referred to as Group B Streptococcus (GBS), is a Gram-positive, $\beta$-hemolytic, chain-forming coccus. It has been recognized as one of the major causes of pneumonia and meningitis in neonates [1], mastitis in cows [2], and meningoencephalitis in fish [3]. Since 2009, serious piscine GBS infections have occurred among tilapia farms in China, causing high mortality and resulting in considerable economic losses [4]. The isolate GD201008-001, a highly virulent strain isolated from a moribund cultured tilapia in China, was identified and sequenced in our laboratory in 2012 [5]. According to our comparative genomics analysis [6], isolates from cultured tilapia in China have a

\footnotetext{
* Correspondence: lucp@njau.edu.cn; xup@ffrc.cn

'Key Laboratory of Animal Bacteriology, Ministry of Agriculture, Nanjing Agricultural University, Weigang No.1, Nanjing 210095, Jiangsu, China ${ }^{2}$ Key Laboratory of Freshwater Fisheries and Germplasm Resources Utilization, Ministry of Agriculture, Freshwater Fisheries Research Center, Chinese Academy of Fishery Sciences, Wuxi 214081, China
}

very close genomic relationship with the human strain A909. In fact, the genomic content of these isolates cannot be distinguished from that of A909. S. agalactiae A909 was originally isolated from the cerebrospinal fluid of a neonate with meningitis in the USA [7]; therefore, $S$. agalactiae infections among fish in China may represent a zoonotic hazard although further investigations are required to confirm this hypothesis.

As a consequence of the potential economic and ecological impacts on public health, many studies have been conducted on $S$. agalactiae with the aim of identifying virulence factors [8], diagnostic markers $[9,10]$ and treatments [11]. Surface proteins of pathogenic bacteria are known to mediate the key step in the bacteria-host interaction during infection and may represent protective antigens and virulence markers. Thus, identification of $S$. agalactiae surface proteins associated with bacterial infection and a greater understanding of their roles in pathogenicity are required. The pre-absorbed immunoproteomics method (PAIM) was successfully established 
as a useful tool for identifying bacterial surface proteins in our previous study $[12,13]$ and has been applied in Escherichia coli [14] and Campylobacter jejuni [15]. This technique involves the separation of proteins by twodimensional electrophoresis (2-DE) and Western blotting with untreated (hyperimmune rabbit serum) and "pre-absorbed" serum, which is produced from untreated serum using a modified cross-absorption process to remove bacterial surface antigen-specific antibodies. Protein spots that appear in the blot probed with untreated sera, but that are absent in the blot probed with preabsorbed sera, are assumed to be surface proteins.

In this study, we performed PAIM analysis of the piscine S. agalactiae strain GD201008-001 and identified a serine-rich repeat protein (Srr-1), designated XF, among the potential surface proteins. Previous research has indicated that Srr-1 is an important surface protein for adherence and virulence in human S. agalactiae strains $[16,17]$. However, the role of Srr-1 in the pathogenicity of piscine $S$. agalactiae is unknown. To investigate this issue, a mutant strain $(\Delta x f)$ and a complemented strain $(\mathrm{C} \Delta x f)$ were constructed using streptococcus-E. coli shuttle vectors pSET4s [18] and pSET2 [19]. Their growth characteristics, adherence to HEp-2 cell and virulence were evaluated in a zebrafish infection model.

\section{Results and discussion}

\section{Pre-absorbed immunoproteomics on S. agalactiae}

Untreated and pre-absorbed antisera were used to probe 2-DE blots of $S$. agalactiae GD201008-001 cell lysates. The immunoreactive spots observed in immunoblots (Figure $1 \mathrm{~B}$ and $\mathrm{C}$ ) were matched with the corresponding protein spots observed in the stained 2-DE gel (Figure 1A) by comparison using the layer function of Photoshop CS. In order to minimize the proteomics trial error, the experiments were repeated at least in triplicate, and only the highly reproducible spots were selected. One spot, XF, that invariably appeared in the blot probed with untreated serum (Figure $1 \mathrm{AB}$ ), but was less distinct in the blot treated with pre-absorbed serum (Figure $1 \mathrm{AC}$ ) in the triplicate repeat tests, was excised from preparative 2-DE gels, subjected to tryptic digestion, and then analyzed by matrix-assisted laser desorption ionization-time of flight mass spectrometry (MALDI-TOF MS) and the PSORT program. Other spots which were not detected using the pre-absorbed serum in repeat experiments were also excised and analyzed by MALDI-TOF MS; however, these proteins were predicted to be cytoplasmic by the PSORT program (data not shown).

Because of the much greater sensitivity of western blotting, the XF spot was more extensive in these blots than in the paired Coomassie brilliant blue G-250 stained gels. Consequently, low abundance proteins with strong immunoreactivity, such as XF, are strongly stained in western blotting analyses. In addition, the serine-rich repeat proteins of $S$. agalactiae have been reported to be high MW glycoproteins $(300-400 \mathrm{kDa})$ due to glycosylation $[17,20]$, although their genes encode just 654-1,326 amino acids. In the present study, the apparent molecular weight of XF was in the $150 \mathrm{kDa}$ range. It can be speculated that the large disparity between the published reports and our result is due to differences in the degree of glycosylation of serine-rich repeat proteins. Furthermore, one of the limitations of 2-DE is the inability to analyze very large (MW $>200 \mathrm{kDa}$ ) or very small $(\mathrm{MW}<10 \mathrm{kDa})$ proteins [21]. Consequently, fully glycosylated serine-rich repeat proteins $(\mathrm{MW}>300 \mathrm{kDa})$ will not be detected in 2-DE.

\section{The Srr-1 protein from piscine and human S. agalatiae strains}

Spot XF was identified as a cell wall surface anchor family protein (gi|406709853) by MALDI-TOF MS (Additional file 1), also known as a serine-rich repeat protein (Srr-1) [22]. It has been confirmed that Srr-1 is localized on the surface of streptococcal cells and binds to human fibrinogen and keratin [23]. Previous immunoproteomics studies have confirmed that Srr-1 is a highly immunoreactive protein that is reactive with both $S$. agalactiae convalescent guinea pig sera and infected tilapia antisera [24]. The conserved domain architecture retrieval tool of NCBI (Figure 2) showed that the Srr-1 protein has an orthologous counterpart among GBS strains: a long and specialized signal sequence, two extensive serine-rich repeat regions (SRR1, 2) that undergo glycosylation, a fibrinogen-binding domain ( $\mathrm{SdrG})$, which is necessary for binding to human fibrinogen and keratin 4 [23], and a typical LPXTG cell wall anchoring motif. The Srr-1 proteins range in size from 654 aa to 1,326 aa and the C-terminal SAS (T/M) repeat region (SRR2) is responsible for the observed size variation, which has also been reported previously by Samen et al. [16]. Blastp analysis showed that the similarity of the amino acid sequence of Srr-1 proteins from 1 aa to 643 aa (containing a SdrG) is greater than $90 \%$, and $100 \%$ identical among piscine strains GD201008-001 and ZQ0910 and human strains A909 and CJB111. Protein XF contains 974 aa, with a SdrG region between 472-629 aa and a SRR2 region from 698-974 aa. In piscine S. agalactiae SA20-06, the Srr-1 protein is only 654 aa and devoid of SRR2 due to a single base (A) deletion at the position 1,917 bp in its ORF leading to early termination. In the study by Takahashi et al. [25], the predicted secondary structures of Srr proteins from S. agalactiae are similar to those of Srr proteins from other streptococcal species. Using the SWISS-MODEL tool, we found that the predicted secondary structures of Srr-1 proteins among $S$. agalactiae were similar, even when compared with strain SA20-06. 


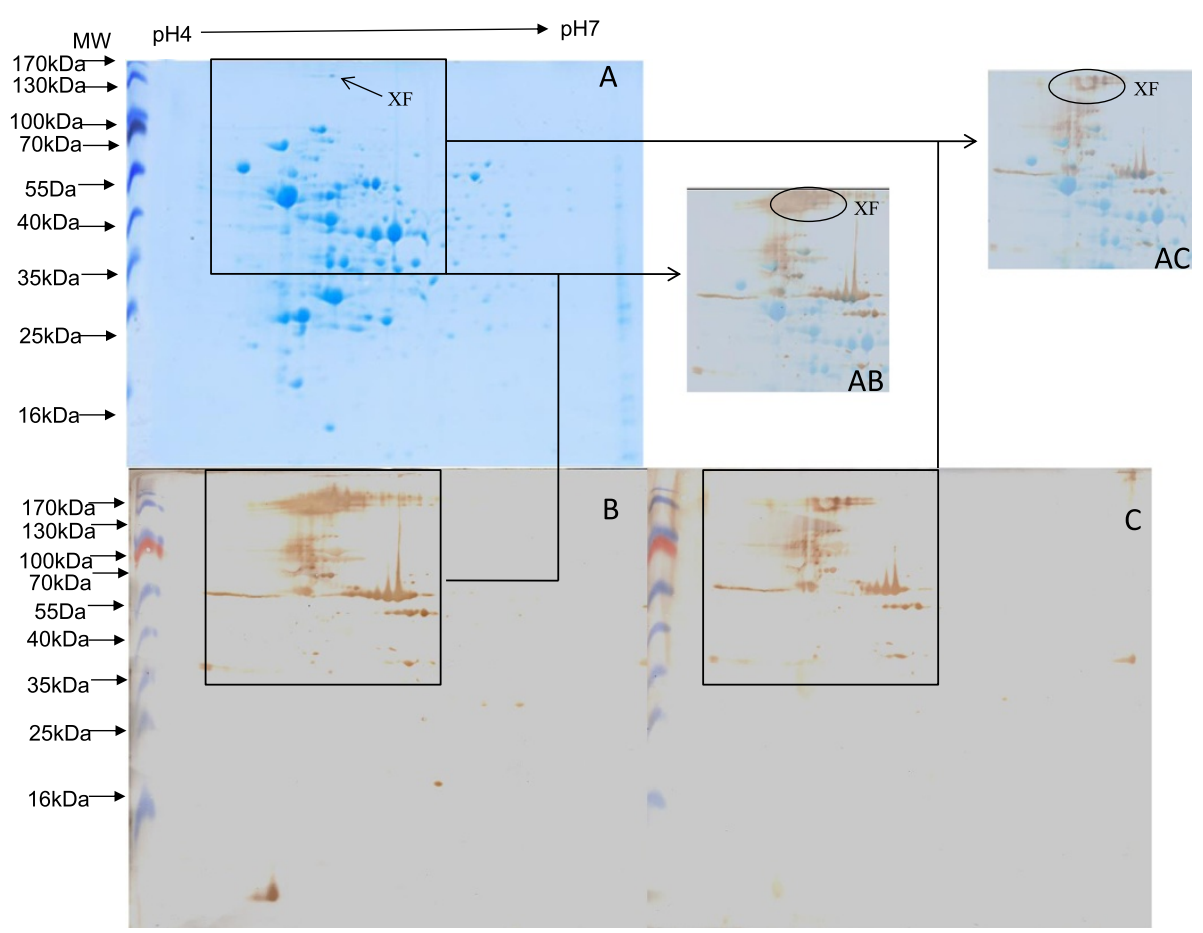

Figure 1 Pre-absorbed immunoproteomics on picsine S. agalactiae GD201008-001. (A) GD201008-001 total cell proteins (pH 4-7, 13 cm), stained with Coomassie brilliant blue G-250. (B) 2-DE blot of GD201008-001 proteins probed with untreated antiserum. (C) 2-DE blot of GD201008-001 proteins probed with "pre-absorbed" antiserum. (AB) Merge of layers A and B. In Photoshop CS, the duplicated gel layer A was used as the background layer and the western blotting layer B as the surface layer in 50\% transparency. (AC) Merge of layers A and C. In Photoshop CS, the duplicated gel layer A was used as the background layer and the western blotting layer C as the surface layer in $50 \%$ transparency.

\section{Construction of the mutant and complementation strains}

To further investigate the function of protein XF in the pathogenesis of piscine $S$. agalactiae, the plasmids pSET4s-XF and pSET2-C $\Delta x f$ were constructed as $x f$ gene deletion (Figure 3) and complementation mutants, respectively.

The pSET4s-XF and pSET2-C $\Delta x f$ plasmids were verified to be correct by diagnostic restriction enzyme digestion with SalI/SmaI and BamHI/PstI, respectively (Figure 4A). The mutant strains $\Delta x f$ and $C \Delta x f$ were confirmed by PCR (Figure 4B) and real-time PCR (Figure 5). The primers XF A and D amplified a 1,377 bp fragment of the $\Delta x f$, while no product was generated from the parental and complementation templates. The XF1/XF2 primers amplified a $289 \mathrm{bp}$ product from the parental GD201008-001 and C $\Delta x f$ templates while no product was obtained from the $\Delta x f$ template. All the PCR products obtained had the expected fragment sizes (Figure $4 \mathrm{~B}$ ) and in correct sequence.

To confirm that the mutant has no effect on the transcription of bilateral genes, qRT-PCR assays was performed to detect the expression of $x f$ gene, $x f$ upstream and downstream ORF genes in the parental, mutant and complementation strains. Data for the three strains were normalized to the expression of the GAPDH gene using the $2^{-\Delta \Delta C T}$ method. The transcription levels of $x f$ gene in the $\Delta x f$ were below the level of detection for this assay and there were no significant differences in $x f$ upstream and downstream gene transcription levels between $\Delta x f$ and $C \Delta x f(P>0.05$; Figure 5$)$. These results indicated the successful construction of an isogenic knockout mutant of $x f$ and complementation of $\Delta x f$, with no effects on the transcription of bilateral genes. The transcription level of $x f$ in the C $\Delta x f$ strain replicated from plasmid pET-2 was higher than that replicated from the genome of the wild-type strain (Figure 5) due to the increased plasmid copy number compared to the genome replication number, with each new cell acquiring at least one copy of the plasmid as the cell divides. Moreover, the plasmid pET-2 was constructed from pSSU1, a native plasmid isolated from S. suis DAT1 [19] and belonging to the pMV158 plasmid family [26]. Lorenzo-Diaz et al. showed that the number of copies of the pMV158 vector was lower in enterococci (around 17 copies per genome equivalent) than that in pneumococci (around 30 copies) [27].

\section{Growth characteristics of the mutant and complementation strains}

Before studying the effect of XF inactivation on the pathogenesis of S. agalctiae in vivo, it is important to 


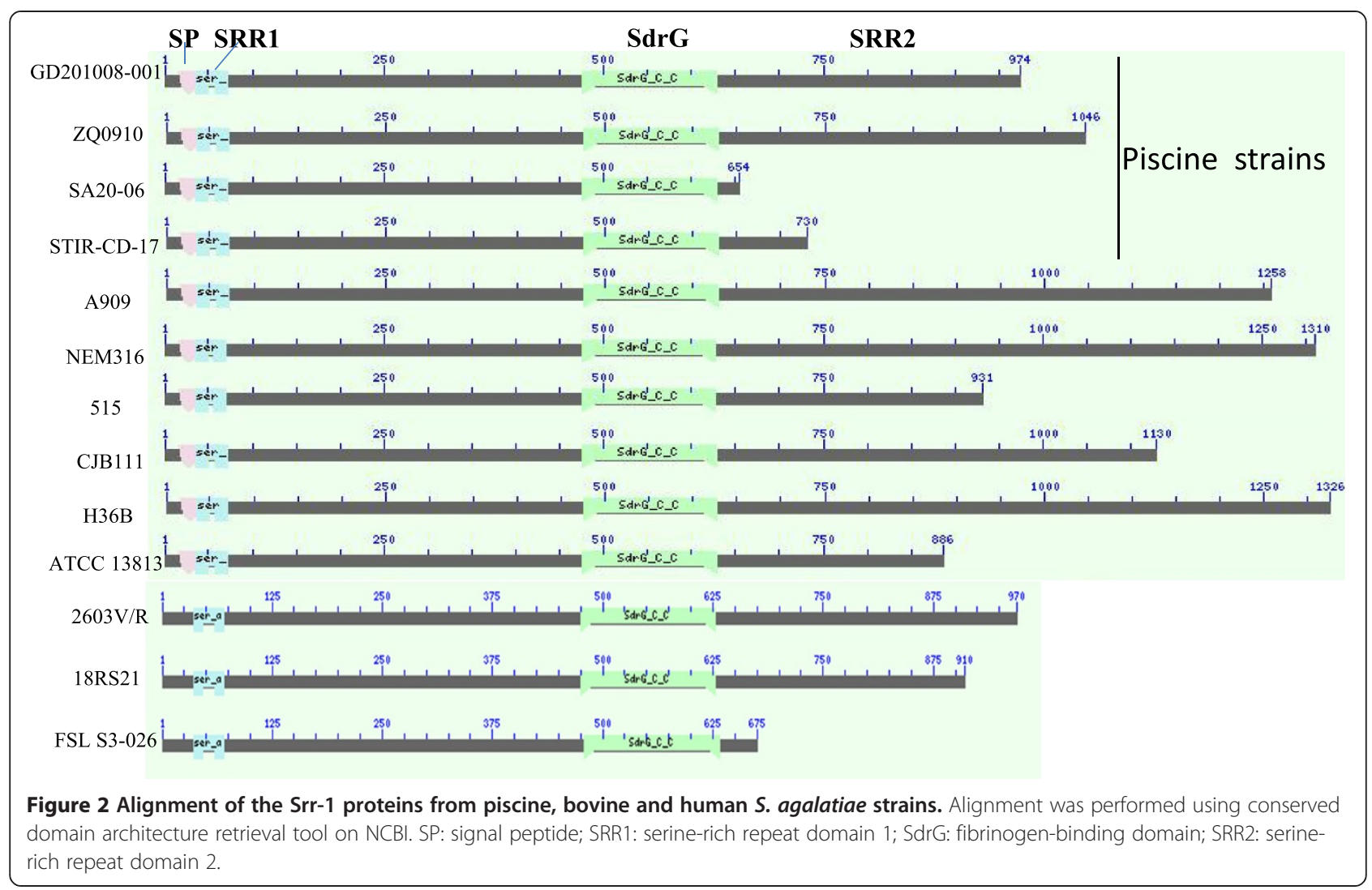

characterize the growth characteristics of the parental, mutant and complementation strains in vitro. The $\mathrm{OD}_{600}$ of cultures of the GD201008-001 and $\Delta x f$ strains in TSB broth and C $\Delta x f$ in TSB broth containing $100 \mu \mathrm{g} / \mathrm{mL}$ spectinomycin at $37^{\circ} \mathrm{C}$ were determined. In addition, the growth curves of these strains at $28^{\circ} \mathrm{C}$ were also determined for evaluation of the $\mathrm{LD}_{50}$ values in a zebrafish infection model at $28^{\circ} \mathrm{C}$. As shown in Figure 6, there were no significant differences in the growth curves of the wild-type, $\Delta x f$ and $C \Delta x f$ strains at the same temperature. The bacterial population reached a peak during the logarithmic growth phase after $6 \mathrm{~h}$ at $37^{\circ} \mathrm{C}$ and after $10 \mathrm{~h}$ at $28^{\circ} \mathrm{C}$. Moreover, no differences in shape and chain formation were observed between the strains following Gram staining (data not shown).

\section{Contribution of XF to adhesion and invasion in vitro}

To investigate the importance of XF for bacterial adherence to, and invasion of host cells, the human epithelial cell line HEp-2 was infected in vitro with the parental, mutant and complementation strains under the same conditions in triplicate repeat tests. As shown in Figure 7, the adherence of the GD201008-001 wild-type strain to HEp- 2 cells was only $0.4 \%$ with a multiplicity of infection

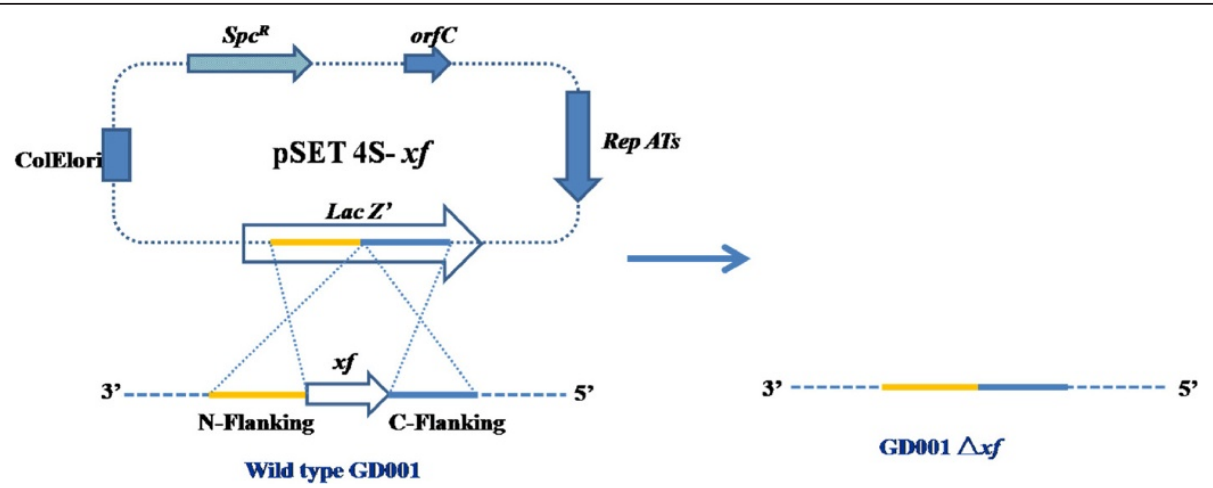

Figure 3 Schematic representation of construction of $x f$ mutant in S. agalactiae GD201008-001 by allelic exchange. Representation of the chromosomal structures before (left) and after (right). 


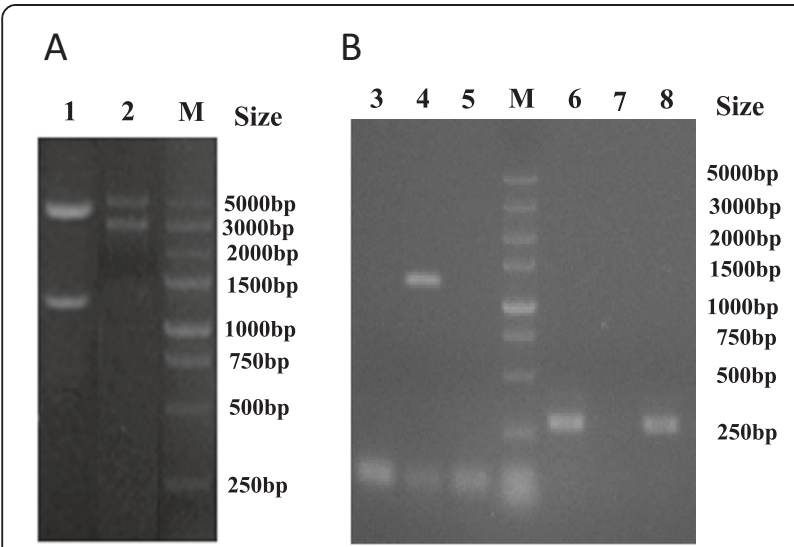

Figure 4 Restriction enzyme digestion identification of recombinant plasmids $(A)$ and PCR confirmation of knockout

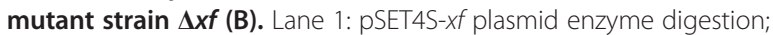
Lane 2: PSET2-C $\Delta x f$ plasmid enzyme digestion; Lane 3: primers XF A/D, GD201008-001; Lane 4: primers XF A/D, $\Delta$ XF; Lane5: primers XF

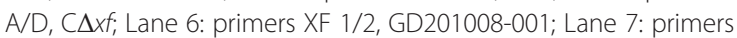
XF1/2, $\Delta x f_{;}$Lane 8: primers XF 1/2, C $\Delta x f_{;}$M: Marker DL5000.

(MOI) of 10 and there were no significant differences in the adherence to HEp-2 cells among the GD201008001, $\Delta x f$ and $C \Delta x f$ strains $(P>0.05)$. In the invasion assays, the parental strain GD201008-001 displayed poor invasion of HEp-2 cells $(<0.0005 \%)$ and there were no significant differences in corresponding activity exhibited by the $\Delta x f$ and $C \Delta x f$ strains (data not shown). These results indicate that piscine $S$. agalactiae GD201008-001 has weak capacity for adherence to and invasion of HEp-2 cells. Thus, XF has almost no effect on bacterial adherence and invasion to HEp-2 cells under the chosen experimental conditions.

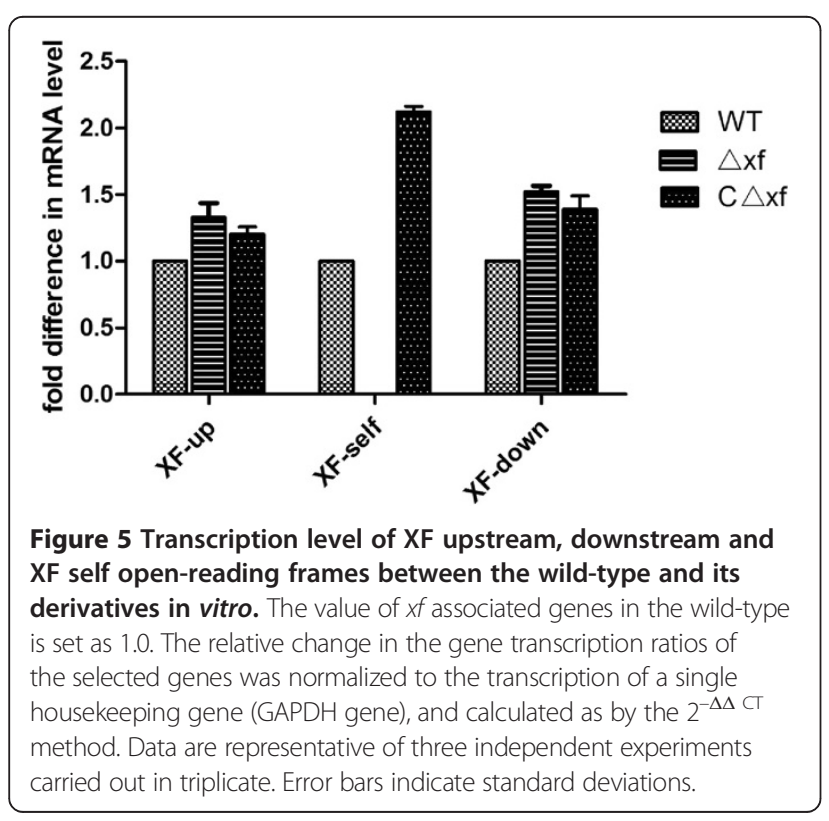

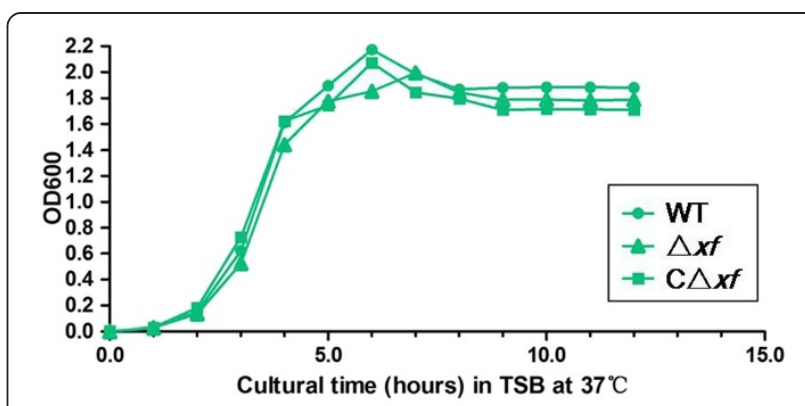

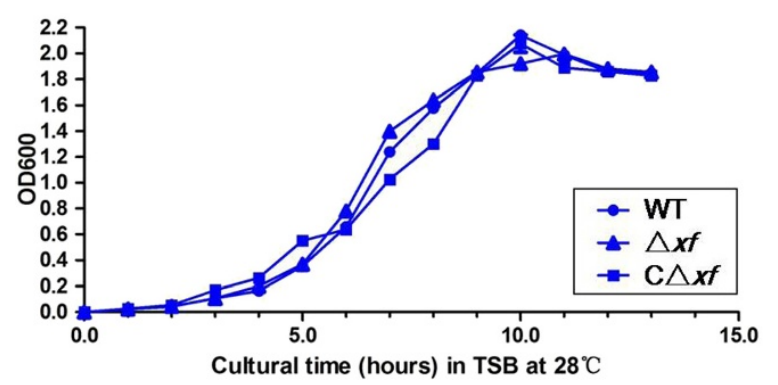

Figure 6 Growth curve of S. agalactiae GD201008-001 and its derivatives at $37^{\circ} \mathrm{C}$ and $28^{\circ} \mathrm{C}$.

The study by Samen et al. showed that Srr- 1 could not mediate bacterial invasion into HEp-2 cells, but promoted adherence of the S. agalactiae human strain 6313 to HEp-2 cells [16]. Compared with our results, Srr-1 showed a different effect on adherence to Hep-2 cells between human and piscine strains. Since piscine S. agalactiae GD201008-001 showed only weak adhesion capability to HEp-2 cells, this cell line may not the best choice for investigation of the cell adhesion capacity of piscine $S$. agalactiae. The contribution of XF to adhesion and invasion in vitro was not clearly reflected under these experimental conditions; therefore, development of an

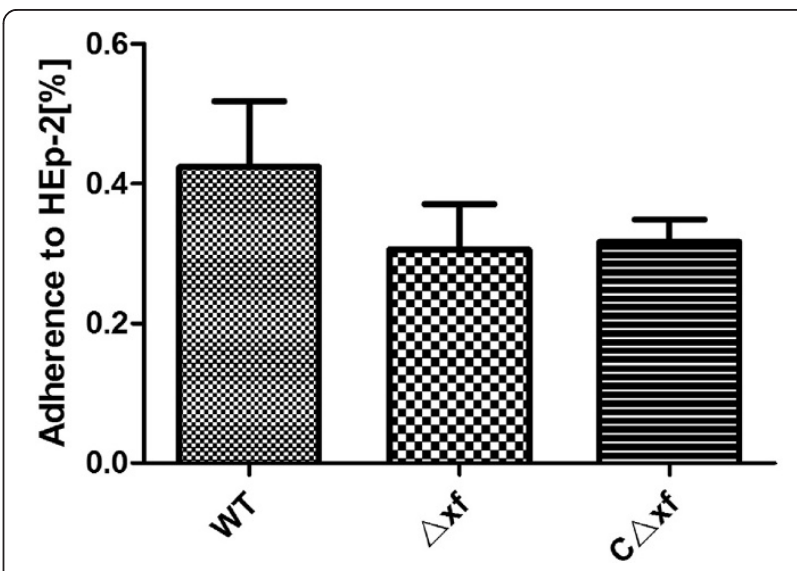

Figure 7 Effects of $x f$ on S. agalactiae adherence to HEp-2 cells (MOI: 10). Data are representative of three independent experiments carried out in triplicate. Error bars indicate standard deviations. 
alternative host cell, such as a tilapia epithelial cell line, is required for more accurate evaluation of the adhesion capability of piscine bacteria in vitro. It can be speculated that the poor capacity of the piscine strain for adherence to and invasion of HEp-2 cells is the result of the loss of some genes nonessential genes involved in adherence to human cells during adaptation to the fish host. For example, there are no $l m b$ or $s c p B$ genes derived from the genomes of piscine $S$. agalactiae strains in the NCBI $\mathrm{nr}$ database to date [28]. These two genes encode two important adhesins in human strains: laminin-binding protein and C5a peptidase, respectively $[29,30]$.

\section{Virulence of wild-type and $\Delta x f$ strains in a zebrafish infection model}

The zebrafish infection model was used to estimate the effect of the XF mutation on $S$. agalactiae virulence. This model has already been established for multiple streptococcal infections, including Streptococcus pyogenes [31], Streptococcus pneumonia [31], Streptococcus suis [32] and Streptococcus agalactiae [33]. Zebrafish have many advantages for this purpose, such as the existence of both innate and adaptive immune systems, short generation time, ease of breeding large numbers in the laboratory and suitability for large-scale genetic screens. Zebrafish were injected intraperitoneally (i.p.) with either the parental GD201008-001, $\Delta x f$ or C $\Delta x f$ strains at various doses in triplicate repeat tests. The mortality of zebrafish was observed within 7 days post-challenge. As shown in Figure 8, the average $\mathrm{LD}_{50}$ values determined in triplicate assays were $2.3 \times 10^{2} \mathrm{CFU} /$ zebrafish for the wild-type strain, $8.6 \times 10^{2} \mathrm{CFU} /$ zebrafish for $\Delta x f$, and $3.3 \times 10^{2} \mathrm{CFU} /$ zebrafish for $C \Delta x f$. The $50 \%$ lethal dose of $\Delta x f$ was increased (4fold) compared with that of the parental strain while that

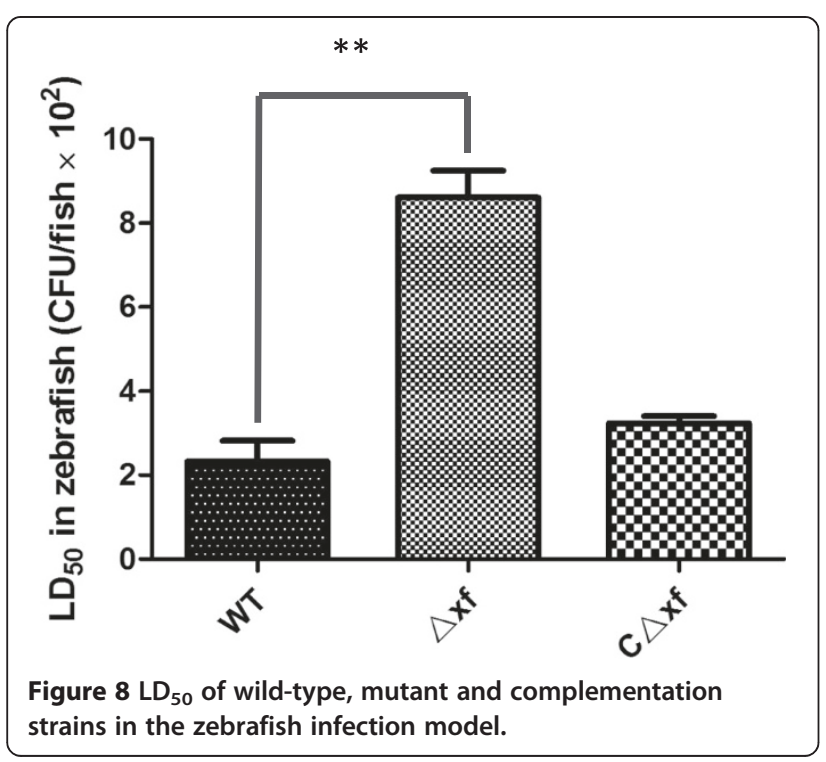

of the complementation strain C $\Delta x f$ was restored to 0.4fold higher than that of the mutant strain $\Delta x f$. These results indicate a significant reduction in the virulence of $S$. agalactiae in the $\Delta x f$ mutant strain $(P<0.05)$.

\section{Conclusions}

In this study, a serine-rich repeat protein family 1 (Srr1), designated XF, was identified by PAIM in the piscine S. agalactiae isolate GD201008-001. The effect of the XF protein on the virulence of piscine $S$. agalactiae in zebrafish was significant despite its low adherence to the human epithelial cell line Hep-2. In conclusion, our study indicates the involvement of Srr-1 in the virulence of $S$. agalactiae in fish.

\section{Methods}

\section{Bacterial strains, plasmids and growth conditions}

Streptococcus agalactiae strain GD201008-001 was isolated from a moribund cultured tilapia with meningoencephalitis in the Guangdong Province of China in 2010 [5]. The strain was grown in trypticase soy broth (TSB, Oxoid) at $37^{\circ} \mathrm{C}$. Escherichia coli DH5 $\alpha$ was supplied by Tiangen (Beijing, China). The streptococcus-E.coli shuttle vectors pSET4s and pSET2 were donated by Dr. Takamatsu from the Laboratory of Molecular Bacteriology, National Institute of Animal Health, Ibaraki, Japan. E. coli strains were maintained in Luria-Bertani (LB) broth at $37^{\circ} \mathrm{C}$. Antibiotics (Sigma) were added to culture media as required at the following concentrations: ampicillin, $50 \mu \mathrm{g} /$ $\mathrm{mL}$ for E. coli; spectinomycin, $100 \mu \mathrm{g} / \mathrm{mL}$ for S. agalactiae, $50 \mu \mathrm{g} / \mathrm{mL}$ for $E$. coli. Bacteria were stored as frozen cultures at $-20^{\circ} \mathrm{C}$ in either TSB or LB, containing $25 \%(\mathrm{v} / \mathrm{v})$ glycerol. All the strains and plasmids used in this study were showed in Table 1.

\section{Pre-absorbed immunoproteomics method Precipitation of bacterial proteins}

GD201008-001 total cell protein precipitations were performed as described previously [24]. Briefly, $30 \mathrm{~mL}$ exponential growth cultures were centrifuged at 10, $000 \times g$ for $15 \mathrm{~min}$ at $4^{\circ} \mathrm{C}$ and washed twice in PBS. Pellets were resuspended in Mutanolysin working buffer (30 mM Tris- $\mathrm{HCl}, 3 \mathrm{mM} \mathrm{MgCl}$, 25\% sucrose) containing 125U/ $\mathrm{mL}$ Mutanolysin (Sigma) and incubated for 90 minutes at $37^{\circ} \mathrm{C}$. The components of solution B (7 M urea, $2 \mathrm{M}$ thiourea, 4\% CHAPS, and 65 mM DTT; GE Healthcare) were added directly into the mixed solution. The turbid solution gradually became transparent and was then sonicated in an ice bath for 50 cycles of $5 \mathrm{~s}$ on/10 s off at $100 \mathrm{~W}$. After $30 \mathrm{~min}$ incubation at $25^{\circ} \mathrm{C}$, unbroken cells was removed by centrifugation at $10,000 \times \mathrm{g}$ for $15 \mathrm{~min}$ at $4^{\circ} \mathrm{C}$. Proteins in the supernatant were precipitated in $10 \%$ pre-chilled trichloroacetic acid (TCA) and incubated in ice-water for $30 \mathrm{~min}$. After centrifugation 
Table 1 Characteristics of bacterial strains, plasmids and primers used in this study

\begin{tabular}{|c|c|c|}
\hline $\begin{array}{l}\text { Strains, plasmids and } \\
\text { primer }\end{array}$ & Characteristics & References \\
\hline GD201008-001 & Virulent strain of piscine S.agalactiae isolated in China & $\begin{array}{l}\text { Collected in our } \\
\text { laboratory }\end{array}$ \\
\hline$\Delta x f$ & Mutation in xf gene of GD201008-001 & In this study \\
\hline$C \Delta x f$ & Complemented strain of $\Delta x f_{;} S p c^{R}$ & In this study \\
\hline E. coli DH5a & Cloning host for maintaining the recombinant plasmids & Invitrogen \\
\hline \multicolumn{3}{|l|}{ Plasmids } \\
\hline pSET4s vector & Temperature-sensitive Streptococcus-E. coli shuttle vector pSET4s; 4506 bp; Spc ${ }^{R}$ & [18] \\
\hline pSET2 vector & Streptococcu- E. coli shuttle vector pSET2; 5016 bp; Spc $c^{R}$ & [19] \\
\hline \multicolumn{3}{|l|}{ Primers } \\
\hline XF A & CGCGTCGACGAAGTTGTAAAGCGTTTG & In this study \\
\hline$X F B$ & TTTGACATGTTCCTCC, amplifies the flank sequence upstream xf coding sequence (665 bp) & \\
\hline XF C & GGAGGAAACATGTCCAAAAAAGACTAAACCTACTIT & \\
\hline XF D & $\begin{array}{l}\text { TCCCCCGGGCCGAACGATTATACAT; amplifies the flank sequence downstream xf coding } \\
\text { sequence ( } 712 \text { bp) }\end{array}$ & \\
\hline CXF F & AAACTGCAGACACAACTCCTTTCACTCATT & \\
\hline CXFR & $\begin{array}{l}\text { CGCGGATCCGGCTITTACAAACTTCTACA; amplifies the structural gene of the } x f \text { gene, } \\
\text { including its own promoter ( } 3407 \mathrm{bp} \text { ) }\end{array}$ & \\
\hline XF 1 & AATGATTGCAAGCGATA & \\
\hline$X F 2$ & GTTAACAGAAGCGATTGA; amplifies the fragment inside the $x f$ gene(289bp) & \\
\hline \multicolumn{3}{|l|}{ RT-PCR Primers } \\
\hline GAPDH-F & GATGACTACTATCCACGCATACAC & In this study \\
\hline GAPDH-R & TGCAGCACCAGTTGAGTTAG & \\
\hline XF up F & CCTGTGTTGGAGTGAAGATAGAG & \\
\hline XF up R & CCAGCAGCCAAGAAAGTAGATA & \\
\hline XF self $F$ & CTTGCAGAACAAACGGAAGTG & \\
\hline XF self $R$ & TGAGGCTGACTCTGACATAGA & \\
\hline XF down F & CTCGTTCTTCTGTCTATCGTCTG & \\
\hline XF down $R$ & ATGCGATATTCGTCACCTACAA & \\
\hline
\end{tabular}

at $10,000 \times \mathrm{g}$ for $10 \mathrm{~min}$ at $4^{\circ} \mathrm{C}$, the pellet was resuspended in $10 \mathrm{~mL}$ of pre-chilled acetone and washed twice. The final pellet was dried in air.

\section{Isoelectric focusing (IEF)}

IEF was performed using the Ettan IPGphor-3 IEF system (GE Healthcare) with $13 \mathrm{~cm}$ (Immobiline DryStrip, pH 4-7; GE Healthcare) gel strips. Prior to rehydration, the precipitated proteins were treated using the 2-D Clean-up Kit (GE Healthcare) to remove contaminants that interfere with electrophoresis. IPG strips were rehydrated overnight at room temperature (RT) with rehydration solution (7 M urea, $2 \mathrm{M}$ thiourea, 2\% CHAPS, 0.2\% DTT, 0.5\% IPG buffer (pH 4-7), and 0.002\% Bromophenol blue. Each strip was loaded with $200 \mu \mathrm{g}$ of protein and IEF was carried out at $20^{\circ} \mathrm{C}$ for $12 \mathrm{~h}$ (maximum voltage of $8,000 \mathrm{~V}$, maximum current of $50 \mu \mathrm{A}$ / IPG strip, total 28,000 Vh).

\section{D SDS-PAGE}

Prior to 2D SDS-PAGE, each IPG strip was washed in equilibration buffer 1 (375 mM Tris- $\mathrm{HCl}$ pH 8.8, $6 \mathrm{M}$ urea, $2 \%$ SDS, $2 \%$ DTT) for 15 min followed by equilibration buffer 2 (375 mM Tris- $\mathrm{HCl}$ pH 8.8, $6 \mathrm{M}$ urea, 2\% SDS, 2.5\% iodoacetamide) for $15 \mathrm{~min}$. Each IPG strip plus a SDSPAGE Molecular Weight Standard (Invitrogen) was loaded on a homogeneous $12 \%$ polyacrylamide gel and sealed with $1 \%$ agarose. Electrophoresis was performed at $15^{\circ} \mathrm{C}$ with an initial voltage of $110 \mathrm{~V}$ for $30 \mathrm{~min}$, followed by $220 \mathrm{~V}$ until the tracking dye reached the gel bottom. All gels were stained with Coomassie brilliant blue G-250 according to the manufacturer's instructions (GE Healthcare). Each 2D IEF/SDS-PAGE experiment was repeated three times.

Preparation of hyperimmune sera and "pre-absorbed" sera Rabbits were first confirmed to be negative for $S$. agalactiae antibodies using an indirect enzyme-linked 
immunosorbent assay (ELISA) developed in-house. Subsequently, these rabbits were immunized with formaldehyde-inactivated S. agalactiae GD201008-001 using Montanide ISA 206 VG (SEPPIC Co. Ltd.) as the adjuvant. Two doses of $1.0 \times 10^{9}$ cells/rabbit were administered by intramuscular injections at 3-week intervals. Sera from immunized rabbits were collected before the first and after the second immunizations. The antibody titers of the sera were evaluated by indirect ELISA.

The absorption protocol used was as described by Mittal et al. [34]. Briefly, exponential cultures of $S$. agalactiae GD201008-001 were centrifuged at 3,000 $\times \mathrm{g}$ for $15 \mathrm{~min}$ at $4^{\circ} \mathrm{C}$, and then washed twice in PBS. A total of $1.0 \times 10^{9}$ bacteria were suspended in $100 \mu \mathrm{L}$ of GD201008-001 hyperimmune serum, incubated for $2 \mathrm{~h}$ at $37^{\circ} \mathrm{C}$, and then overnight at $4^{\circ} \mathrm{C}$. Bacteria were pelleted by centrifugation at $10,000 \times \mathrm{g}$ for $30 \mathrm{~min}$. The supernatant was collected and used as the "pre-absorbed" serum in Western blotting analyses.

\section{Western blotting}

The protein samples from each SDS-PAGE gel were transferred onto PVDF membranes (GE Healthcare) using a semi-dry blotting apparatus (TE77, GE Healthcare) for $2 \mathrm{~h}$ at $0.65 \mathrm{~mA} / \mathrm{cm}^{2}$. The membrane was then blocked with $5 \%(\mathrm{w} / \mathrm{v})$ skimmed milk in $50 \mathrm{mM}$ Tris$\mathrm{HCl}$ buffer (pH 7.4) containing $0.05 \%$ Tween 20 (TBST) for $2 \mathrm{~h}$ at RT. The blocked membrane was then incubated with GD201008-001 hyperimmune serum or "preabsorbed" serum (1: 200 dilution) for $2 \mathrm{~h}$ at RT and washed three times with TBST (10 min per wash). The membrane was incubated with HRP-goat anti rabbit IgG (1:10,000 dilution; Boster) at RT for $1 \mathrm{~h}$, washed three times with TBST, and developed by adding DAB (Tiangen) until the optimum color was obtained. Western blotting was repeated in triplicate.

\section{MALDI-TOF MS and database searching}

Proteins identified from the 2-DE blots as potential surface proteins were excised from duplicate SDS-PAGE gels and subjected to in-gel trypsin digestion and MALDI-TOF MS analysis (TOF Ultraflex II mass spectrometer, BrukerDaltonics). Peptide mass fingerprinting data (PMF) were analyzed using the MASCOT server (www.matrixscience.com). MASCOT searches were used to identify significant peptides and for determination of the combined peptide scores. The extent of sequence coverage, number of matched peptides, and the score probability obtained from the PMF data were all used to validate protein identification. Low-scoring proteins were either verified manually or rejected.

\section{Bioinformatics analysis}

Sequences of the identified proteins were searched using the BLASTX server (http://www.ncbi.nlm.nih.gov/BLASTX/) to identify homologous sequences. The PSORT server (http:// www.psort.org/) program was used to predict protein subcellular localizations.

\section{Construction of the mutant $\Delta x f$}

Two DNA fragments flanking the $x f$ gene were amplified by PCR from the genome of GD201008-001 with the primers XF A/B and primers XF C/D with incorporated SalI and SmaI restriction enzyme sites, respectively in primers A and D (Table 1). The resulting PCR products were mixed in equal amounts and subjected to a crossover PCR to generate a single PCR product. The crossover PCR product and the temperature-sensitive streptococcus- $E$. coli shuttle vector pSET4s were digested with appropriate enzymes, ligated and used to transform E. coli DH5 $\alpha$. The resulting pSET4S-XF plasmid was introduced into competent $S$. agalactiae GD201008-001 cells by electroporation at $2.35 \mathrm{KV}, 200 \Omega$ and $25 \mu \mathrm{F}$.

The procedure for the selection of mutants generated by allelic exchange via double-crossover was performed as described previously [18] with some modifications. In the presence of the appropriate antibiotics, the temperaturesensitive plasmid can be propagated in $E$. coli at $37^{\circ} \mathrm{C}$, while its replication is blocked in Streptococcus above $37^{\circ} \mathrm{C}$. After electroporation, $S$. agalactiae containing the pSET4S-xf plasmid were first grown at $28^{\circ} \mathrm{C}$ in THY (THB supplemented with $2 \%$ yeast extract) for $4 \mathrm{~h}$ to recover, and then cultured in THY containing $100 \mu \mathrm{g} / \mathrm{mL}$ spectinomycin to mid-logarithmic growth phase. The pSET4S- $x f$ plasmid in $S$. agalactiae duplicated well in the appropriate antibiotics at $28^{\circ} \mathrm{C}$. The cells were then diluted and plated on TSB containing $100 \mu \mathrm{g} / \mathrm{mL}$ spectinomycin and cultured at $37^{\circ}$ C. When the temperature increased from $28^{\circ} \mathrm{C}$ to $37^{\circ} \mathrm{C}$, the replication of this vector in S. agalactiae is blocked immediately and the bacteria without the pSET4S- $x f$ plasmid are killed by spectinomycin. Thus, at the non-permissive temperature $\left(37^{\circ} \mathrm{C}\right)$ under antibiotic pressure, most of bacteria were destroyed and only a few survived by integrating the pSET4S- $x f$ plasmid genes into their own chromosome by allelic exchange via single crossover recombination. The single bacterial colony on the $100 \mu \mathrm{g} / \mathrm{mL}$ spectinomycin TSB plate cultured at $37^{\circ} \mathrm{C}$ was transferred to $28^{\circ} \mathrm{C}$ in TSB liquid without antibiotics. These conditions forced loss of the spectinomycin-resistance gene in their chromosome by allelic exchange. During the complete procedure, the chromosomal $x f$ gene of $S$. aglactiae was replaced by a total double-crossover event (Figure 3). The identity of the correct $\Delta x f$ mutants was confirmed by PCR using XF A/D, $\mathrm{XF} 1 / 2$ primers and DNA sequencing of the PCR product.

\section{Functional complementation of $\Delta \mathbf{x f}$}

The $x f$ gene, including its own promoter, was amplified from the chromosomal DNA of GD201008-001 by PCR using the primers C XF F and C XF R (Table 1). The PCR 
product was cut with BamHI/PstI and ligated into the BamHI/PstI digested pSET2 [19] to generate the recombinant plasmid pSET2-C $\Delta x f$. The identity of the correct pSET2-C $\Delta x f$ vector was confirmed by sequencing using the primers $\mathrm{C}$ XF F and C XF R. The pSET2-C $\Delta x f$ plasmid was then used to electrotransform the $\Delta x f$ mutant to create the complemented strain of $\Delta \mathrm{XF}$ (designated $\mathrm{C} \Delta x f$ ) on THB agar under spectinomycin selection pressure.

\section{Quantitative real-time reverse transcription PCR (qRT-PCR)}

$S$. agalactiae strains GD201008-001, $\Delta x f$, C $\Delta x f$ were cultured overnight in TSB at $37^{\circ} \mathrm{C}$ and isolated RNA with an E.Z.N.A. ${ }^{\text {TM }}$ Bacterial RNA isolation kit (OMEGA, Beijing, China). The cDNA synthesis was performed using the PrimeScript $^{\text {Tx }}$ RT reagent kit with DNA eraser (TaKaRa, Dalian, China) according to the manufacturer's instructions. The mRNA levels were measured using two-step relative qRT-PCR. The glyceraldehyde-3-phosphate dehydrogenase (GAPDH) housekeeping gene was amplified as an internal control. The specific primers used for the various RT-PCR assays are listed in Table 1 . The SYBR Green PCR method was performed using the $A_{c e Q}{ }^{\mathrm{ma}} \mathrm{qPCR}$ SYBR $^{\mathrm{TM}}$ Green Master Mix (Vazyme Biotech Co., Ltd.). Reactions were carried out in triplicate and repeated three times. An ABI 7300 RT-PCR system (Applied Biosystems) was used for relative qRT-PCR. Dissociation analysis of amplification products was performed at the end of each PCR to confirm that only one PCR product was amplified and detected. The comparative cycle threshold method ( $2^{-\Delta \Delta C T}$ method) [35] was used to analyze the mRNA levels.

\section{Growth characteristics of mutant strains}

The wild-type strain GD201008-001, mutant strain $\Delta x f$ and the complementation strain $\mathrm{C} \Delta x f$ were separately inoculated into flasks containing $100 \mathrm{~mL}$ TSB media, and incubated at $37^{\circ} \mathrm{C}$ and $28^{\circ} \mathrm{C}$. Samples of culture were monitored at $1 \mathrm{~h}$ intervals for $13 \mathrm{~h}$ using a spectrophotometer (Bio-Rad, USA) at an absorbance of $600 \mathrm{~nm}$. The un-inoculated TSB served as the blank control. The experiments were repeated twice.

\section{Cell adherence assay}

The cell adherence and invasion assay was performed as previously described [36]. Briefly, the human laryngeal cell line HEp-2 cells was cultured in RPMI 1640 media (Invitrogen, USA) in 24-well tissue culture plates, supplemented with $10 \%$ fetal calf serum (FCS), and maintained at $37^{\circ} \mathrm{C}$ in a $5 \% \mathrm{CO}_{2}$ humidified incubator. The wild-type strain GD201008-001, mutant strain $\Delta x f$ and complementation strain $\mathrm{C} \Delta x f$ were cultured separately in TSB medium to the logarithmic phase and diluted to the appropriate density to give a MOI of 10 in RPMI 1640 medium (without FCS). The monolayers were washed by RPMI 1640 medium (without FCS) and incubated with diluted bacteria. The plates were centrifuged at $800 \times \mathrm{g}$ for $10 \mathrm{~min}$ at RT before incubation for $2 \mathrm{~h}$ at $37^{\circ} \mathrm{C}$. After incubation, the medium was removed from each well and monolayers were washed five times using PBS ( $\mathrm{pH} 7.4$ ). For the cellular invasion assay, the extracellular bacteria were eliminated by additional incubation of the monolayers with experimental medium containing gentamicin $(100 \mu \mathrm{g} / \mathrm{mL})$ for $120 \mathrm{~min}$ at $37^{\circ} \mathrm{C}$ and the plate was washed five times using PBS. HEp-2 cells were detached from plates by treatment with $100 \mu \mathrm{L}$ of $0.25 \%$ trypsin- $-0.1 \%$ EDTA prepared in PBS ( $\mathrm{pH} 7.4$ ). The cells were lysed by the addition of $900 \mu \mathrm{L} \mathrm{ddH}_{2} \mathrm{O}$ and appropriate dilutions were plated on TSB agar plates for enumeration of the bacteria adhering or invading to HEp-2 cells. Negative control wells containing only cells were used in all experiments. All assays were performed in triplicate and repeated at least three times.

\section{Animal experiments}

The feeding and care of zebrafish supplied by the Pearl River Fishery Research Institute of the Chinese Academy of Fishery Science were performed as described by Neely et al. [31]. The virulence of streptococcus was evaluated in a zebrafish model as described previously [32]. Specifically, $50 \%$ lethal doses $\left(\mathrm{LD}_{50}\right)$ were ascertained to determine the differences in virulence between the wild-type and mutant strains [33]. Prior to inoculation of fish, cultures (S. agalactiae strains GD201008-001, $\Delta x f$ and $C \Delta x f$ ) were collected, cultured at $37^{\circ} \mathrm{C}$ to the logarithmic growth phase, washed twice in TSB and adjusted to the appropriate doses (CFU/ fish). Zebrafish were anesthetized with tricaine methanesulfonate (MS-222) (Hangzhou Animal Medicine Factory) at a concentration of $80 \mathrm{mg} / \mathrm{L}$. The bacterial dose (CFU) contained in the injected inoculum was confirmed at the time of infection by plating onto TSB agar. Control fish were injected with TSB. Fifteen fish were used to test each dose. Mortality was monitored until 7 days postinfection. The experiment was repeated three times and the results were used to calculate the $\mathrm{LD}_{50}$ values using the method of Reed and Muench [37]. The experimental animal use license for the zebrafish studies was approved by the Science and Technology Agency of Jiangsu Province (China).

\section{Statistical analyses}

All the statistical analysis was performed using SPSS version 17.0 (SPSS Inc., Chicago, IL). Differences between the mean values among groups was first evaluated by one-way analysis of variance (ANOVA) and then by pairwise comparison of the mean values between the two groups, followed by Tukey's student rank test. Differences with a $P$-value of $<0.05$ were considered significant, and a $P$-value of $<0.001$ was considered highly significant. 


\section{Additional file}

Additional file 1: Table S1. The MALDI-TOF MS and database screening results of XF spot.

\section{Abbreviations}

PAIM: Pre-absorbed immunoproteomic method; Srr: Serine-rich repeat protein; GBS: Group B Streptococcus; MALDI-TOF MS: Matrix-assisted laser desorption ionization-time of flight mass spectrometry;

GAPDH: Glyceraldehyde 3-phosphate dehydrogenase; HEp-2 cell: Human epidermoid cancer cells; MOI: Multiplicity of infection; TSB: Tryptic soy broth; DTT: Dichlorodiphenyltrichloroethane; CHAPS: 3-[(3- cholamidopropyl) dimethylammonio]-1-propanesulfonate; TCA: Trichloroacetic acid; LB: Lysogeny broth; IEF: Isoelectric focusing; SDS-PAGE: Sodium dodecyl sulfate polyacrylamide gel electrophoresis; LD $_{50}$ : 50\% lethal doses; ELISA: Enzyme-linked immunosorbent assay; THB: Todd-Hewitt Broth; RPMI 1640 medium: Roswell Park Memorial Institute1640 medium; CFU: Colony-Forming Units.

\section{Competing interests}

The authors declare that they have no competing interests.

\section{Authors' contributions}

LG carried out all the experiments, participated in the sequence analysis and drafted the manuscript. ZW and LY participated in the design of the study. $Y H, L C$ and XP helped to draft the manuscript. All authors read and approved the final manuscript.

\section{Acknowledgements}

This work was supported by the Open Fund from Key Laboratory of Freshwater Fisheries and Germplasm Resources Utilization (KF201301), the Youth Foundation of Natural Science Foundation of Jiangsu Province, China (BK20140703), the Post-graduate Training Innovation Project from the Education Department of Jiangsu Province, China (CXZZ12_0297), the Special Fund for Public Welfare Industry of Chinese Ministry of Agriculture (2013003041) and the Priority Academic Program Development of Jiangsu Higher Education Institutions (PAPD)

Received: 11 June 2014 Accepted: 17 October 2014

Published online: 26 October 2014

\section{References}

1. Mhalu FS: Infection with Streptococcus agalactiae in a London hospital. J Clin Pathol 1976, 29(4):309-312.

2. Schalm OW, Casselberry NH: Treatment of Streptococcus agalactiae infections with intramammary infusions of penicillin. J Am Vet Med Assoc 1946, 109(837):470-477.

3. Vandamme P, Devriese LA, Pot B, Kersters K, Melin P: Streptococcus difficile is a nonhemolytic group B, type Ib Streptococcus. Int J Syst Bacterio/ 1997, 47(1):81-85.

4. Li L, Wang R, Liang W, Gan X, Huang T, Huang Y, Li J, Shi Y, Chen M, Luo H: Rare serotype occurrence and PFGE genotypic diversity of Streptococcus agalactiae isolated from tilapia in China. Vet Microbiol 2013, 167(3-4):719-724.

5. Liu G, Zhang W, Lu C: Complete Genome Sequence of Streptococcus agalactiae GD201008-001, Isolated in China from Tilapia with Meningoencephalitis. J Bacterio/ 2012, 194(23):6653.

6. Liu G, Zhang W, Lu C: Comparative genomics analysis of Streptococcus agalactiae reveals that isolates from cultured tilapia in China are closely related to the human strain A909. BMC Genomics 2013, 14(1):775.

7. Bray BA, Sutcliffe IC, Harrington DJ: Expression of the MtsA lipoprotein of Streptococcus agalactiae A909 is regulated by manganese and iron. Antonie Van Leeuwenhoek 2009, 95(1):101-109.

8. Lin FP, Lan R, Sintchenko V, Gilbert GL, Kong F, Coiera E: Computational bacterial genome-wide analysis of phylogenetic profiles reveals potential virulence genes of Streptococcus agalactiae. PLOS ONE 2011, 6(4):e17964.

9. Terp MG, Lund RR, Jensen ON, Leth-Larsen R, Ditzel HJ: Identification of markers associated with highly aggressive metastatic phenotypes using quantitative comparative proteomics. Cancer Genomics Proteomics 2012, 9(5):265-273.
10. Morita T, Feng D, Kamio Y, Kanno I, Somaya T, Imai K, Inoue M, Fujiwara M, Miyauchi A: Evaluation of chromIDstrepto $B$ as a screening media for Streptococcus agalactiae. BMC Infect Dis 2014, 14:46.

11. Brnakova Z, Farkasovska J, Godany A: The use of bacteriophages in eliminating polyresistant strains of Staphylococcus aureus and Streptococcus agalactiae. Folia Microbiol (Praha) 2005, 50(3):187-194.

12. Zhang W, Liu G, Tang F, Shao J, Lu Y, Bao Y, Yao H, Lu C: Pre-absorbed immunoproteomics: a novel method for the detection of Streptococcus suis surface proteins. PLOS ONE 2011, 6(6):e21234.

13. Liu G, Zhang W, Lu C: Pre-absorbed immunoproteomics: a novel method for the detection of bacterial surface proteins. Methods Mol Biol 2013, 1061:113-121.

14. Bao Y, Zhai Z, Wang S, Ma J, Zhang W, Lu C: Chaperonin GroEL: a novel phylogenetically conserved protein with strong immunoreactivity of Avian Pathogenic Escherichia coli isolates from duck identified by immunoproteomics. Vaccine 2013, 31(28):2947-2953.

15. Hu Y, Shang Y, Huang J, Wang Y, Ren F, Jiao Y, Pan Z, Jiao XA: A novel immunoproteomics method for identifying in vivo-induced Campylobacter jejuni antigens using pre-adsorbed sera from infected patients. Biochim Biophys Acta 2013, 1830(11):5229-5235.

16. Samen U, Eikmanns BJ, Reinscheid DJ, Borges F: The surface protein Srr- 1 of Streptococcus agalactiae binds human keratin 4 and promotes adherence to epithelial HEp-2 cells. Infect Immun 2007, 75(11):5405-5414.

17. Seo HS, Mu R, Kim BJ, Doran KS, Sullam PM: Binding of glycoprotein Srr1 of Streptococcus agalactiae to fibrinogen promotes attachment to brain endothelium and the development of meningitis. PLOS Pathog 2012, 8(10):e1002947.

18. Takamatsu D, Osaki M, Sekizaki T: Thermosensitive suicide vectors for gene replacement in Streptococcus suis. Plasmid 2001, 46(2):140-148.

19. Takamatsu D, Osaki M, Sekizaki T: Construction and characterization of Streptococcus suis-Escherichia coli shuttle cloning vectors. Plasmid 2001 45(2):101-113.

20. van Sorge NM, Quach D, Gurney MA, Sullam PM, Nizet V, Doran KS: The group B streptococcal serine-rich repeat 1 glycoprotein mediates penetration of the blood-brain barrier. J Infect Dis 2009, 199(10):1479-1487.

21. Bunai K, Yamane K: Effectiveness and limitation of two-dimensional gel electrophoresis in bacterial membrane protein proteomics and perspectives. J Chromatogr B Anal Technol Biomed Life Sci 2005, 815(1-2):227-236.

22. Seifert KN, Adderson EE, Whiting AA, Bohnsack JF, Crowley PJ, Brady LJ: A unique serine-rich repeat protein (Srr-2) and novel surface antigen (epsilon) associated with a virulent lineage of serotype III Streptococcus agalactiae. Microbiology 2006, 152(Pt 4):1029-1040.

23. Sundaresan R, Samen U, Ponnuraj K: Expression, purification, crystallization and preliminary $\mathrm{X}$-ray diffraction studies of the human keratin 4-binding domain of serine-rich repeat protein 1 from Streptococcus agalactiae. Acta Crystallogr Sect F Struct Biol Cryst Commun 2011, 67(Pt 12):1582-1585.

24. Liu G, Zhang W, Lu C: Identification of immunoreactive proteins of Streptococcus agalactiae isolated from cultured tilapia in China. Pathogens Dis 2013, 69(3):223-231.

25. Takahashi Y, Konishi K, Cisar JO, Yoshikawa M: Identification and characterization of hsa, the gene encoding the sialic acid-binding adhesin of Streptococcus gordonii DL1. Infect Immun 2002, 70(3):1209-1218.

26. Takamatsu D, Osaki M, Sekizaki T: Sequence analysis of a small cryptic plasmid isolated from Streptococcus suis serotype 2. Curr Microbiol 2000, 40(1):61-66

27. Lorenzo-Diaz F, Espinosa M: Lagging-strand DNA replication origins are required for conjugal transfer of the promiscuous plasmid pMV158. J Bacteriol 2009, 191(3):720-727.

28. Nur-Nazifah M, Sabri MY, Siti-Zahrah A: Development and efficacy of feed-based recombinant vaccine encoding the cell wall surface anchor family protein of Streptococcus agalactiae against streptococcosis in Oreochromis sp. Fish Shellfish Immunol 2014, 37(1):193-200.

29. Beckmann C, Waggoner JD, Harris TO, Tamura GS, Rubens CE: Identification of novel adhesins from Group B streptococci by use of phage display reveals that $\mathrm{C} 5 \mathrm{a}$ peptidase mediates fibronectin binding. Infect Immun 2002, 70(6):2869-2876.

30. Spellerberg B, Rozdzinski E, Martin S, Weber-Heynemann J, Schnitzler N, Lutticken R, Podbielski A: Lmb, a protein with similarities to the Lraladhesin family, mediates attachment of Streptococcus agalactiae to human laminin. Infect Immun 1999, 67(2):871-878. 
31. Neely MN, Pfeifer JD, Caparon M: Streptococcus-zebrafish model of bacterial pathogenesis. Infect Immun 2002, 70(7):3904-3914.

32. Wu Z, Zhang W, Lu Y, Lu C: Transcriptome profiling of zebrafish infected with Streptococcus suis. Microb Pathog 2010, 48(5):178-187.

33. Patterson H, Saralahti A, Parikka M, Dramsi S, Trieu-Cuot P, Poyart C, Rounioja S, Ramet M: Adult zebrafish model of bacterial meningitis in Streptococcus agalactiae infection. Dev Comp Immunol 2012, 38(3):447-455.

34. Mittal KR, Higgins $R$, Lariviere $S$ : Evaluation of slide agglutination and ring precipitation tests for capsular serotyping of Haemophilus pleuropneumoniae. J Clin Microbiol 1982, 15(6):1019-1023.

35. Livak KJ, Schmittgen TD: Analysis of relative gene expression data using real-time quantitative PCR and the 2(-Delta DeltaC(T)) Method. Methods 2001, 25(4):402-408.

36. Gutekunst H, Eikmanns BJ, Reinscheid DJ: Analysis of RogB-controlled virulence mechanisms and gene repression in Streptococcus agalactiae. Infect Immun 2003, 71(9):5056-5064.

37. Reed $L$, Muench $\mathrm{H}$ : A simple method of estimating fifty percentend points. AmJ Hyg 1938, 27:493-497.

doi:10.1186/s12917-014-0259-7

Cite this article as: Liu et al:: Identification of a virulence-related

surface protein XF in piscine Streptococcus agalactiae by pre-absorbed immunoproteomics. BMC Veterinary Research 2014 10:259.

\section{Submit your next manuscript to BioMed Central and take full advantage of:}

- Convenient online submission

- Thorough peer review

- No space constraints or color figure charges

- Immediate publication on acceptance

- Inclusion in PubMed, CAS, Scopus and Google Scholar

- Research which is freely available for redistribution 